\title{
Bianchi Type-IX Barotropic Fluid Model with Time-Dependent Displacement Vector in Lyra Geometry
}

\section{Raj Bali, Rajendra Vadhwani, and Jagdish Prasad Dhanka}

Department of Mathematics, University of Rajasthan, Jaipur 302004, India

Correspondence should be addressed to Raj Bali, balir5@yahoo.co.in

Received 17 September 2011; Accepted 19 October 2011

Academic Editors: J. Bičák and D. Singleton

Copyright (C) 2012 Raj Bali et al. This is an open access article distributed under the Creative Commons Attribution License, which permits unrestricted use, distribution, and reproduction in any medium, provided the original work is properly cited.

Bianchi Type IX barotropic fluid cosmological model in the frame work of Lyra geometry is investigated. To get the deterministic model of the universe, it is assumed that shear $(\sigma)$ is proportional to expansion $(\theta)$. This leads to $a=b^{n}$, where $a$ and $b$ are metric potentials and $n$ is a constant. To get the results in terms of cosmic time $t$, we have also considered a special case $\gamma=0$ (dust filled universe) and $n=2$. We find that the model starts with a big bang initially and the displacement vector $(\beta)$ is initially large but decreases due to lapse of time. The models $d s^{2}=-\left(T^{6} /\left[3 N^{2} / 20-((1-\gamma) / 4(5 \gamma+7)) T^{8}-(\gamma /(5 \gamma+\right.\right.$ 4)) $\left.\left.T^{6}\right]\right) d T^{2}+T^{4} d X^{2}+T^{2} d Y^{2}+\left(T^{2} \sin ^{2} Y+T^{4} \cos ^{2} Y\right) d Z^{2}-2 T^{4} \cos Y d X d Z$ and $d s^{2}=-d \tau^{2}+$ $[\sqrt{21 / 5} N \sin ((2 / \sqrt{7}) \tau)] d x^{2}+[\sqrt{21 / 5} N \sin ((2 / \sqrt{7}) \tau)]^{1 / 2} d y^{2}+\left([\sqrt{21 / 5} N \sin ((2 / \sqrt{7}) \tau)]^{1 / 2} \sin ^{2} y+\right.$ $\left.[\sqrt{21 / 5} N \sin ((2 / \sqrt{7}) \tau)] \cos ^{2} y\right) d z^{2}-2[\sqrt{21 / 5} N \sin ((2 / \sqrt{7}) \tau)] \cos y d x d z$ have point-type singularity at $T=0$ and $\tau=0$, respectively. The physical and geometrical aspects of the models are also discussed.

\section{Introduction}

Bianchi Type IX space-time is the generalization of FRW model with positive curvature. Bianchi Types cosmological models create more interest in the study, because familiar models like Robertson-Walker model [1], the de-Sitter universe [2], Taub-NUT [3, 4] space times are of Bianchi Type IX space-time. The solutions [3,4] allow expansion, rotation, and shear. Vaidya and Patel [5] have obtained the solution for spatially homogeneous Bianchi Type IX space time and have given a general scheme for the derivation of exact solutions of Einstein's field equations corresponding to a perfect fluid and pure radiation field. Bianchi Type IX space times are also studied by many research workers namely Krori et al. [6], Chakraborty and Nandy [7], Chakraborty [8], and Bali and Upadhaya [9]. 
By geometrizing gravitation, Einstein derived the field equations of general relativity. Weyl [10] developed a theory to geometrize gravitation and electromagnetism inspired by the idea of geometrizing gravitation of Einstein. But Weyl's theory was discarded due to nonintegrability of length of vector under parallel displacement. Lyra [11] modified Riemannian geometry by introducing a gauge function into the structureless manifold. This step removed the main obstackle of Weyl's theory [10] and made length of vector integrable under parallel displacement. Sen [12] investigated an analogue of Einstein's field equation by introducing a new scalar theory of gravitation. Halford [13] pointed out that constant displacement vector $\left(\phi_{\mu}\right)$ in Lyra geometry plays the role of cosmological constant in General Relativity. A number of authors, namely, T. Singh and G. P. Singh [14], Rahman and Bera [15], Rahman et al. [16], Pradhan et al. [17-19], Bali and Chandnani [20, 21], Ram et al. [22], and Bali et al. [23], have investigated cosmological models for different Bianchi space time under different contexts in the frame work of Lyra geometry.

In this paper, we have investigated Bianchi Type IX barotropic fluid cosmological model in the frame work of Lyra geometry. To get the deterministic model, we have assumed that the shear $(\sigma)$ is proportional to expansion $(\theta)$. We have also considered the dust distribution $(p=0)$ model to get the result in terms of cosmic time. We find that the model starts with a big bang initially and expansion decreases as time increases. The displacement vector is initially large but decreases due to lapse of time. The physical and geometrical aspects of the models are also discussed.

\section{The Metric and Field Equations}

We consider Bianchi Type IX metric in the form

$$
d s^{2}=-d t^{2}+a^{2} d x^{2}+b^{2} d y^{2}+\left(b^{2} \sin ^{2} y+a^{2} \cos ^{2} y\right) d z^{2}-2 a^{2} \cos y d x d z
$$

where $a$ and $b$ are functions of $t$-alone.

The energy momentum tensor $\left(T_{i}^{j}\right)$ for perfect fluid distribution is given by

$$
T_{i}^{j}=(\rho+p) v_{i} v^{j}+p g_{i}^{j}
$$

The modified Einstein's field equation in normal gauge for Lyra's manifold obtained by Sen [12] is given by

$$
R_{i}^{j}-\frac{1}{2} R g_{i}^{j}+\frac{3}{2} \phi_{i} \phi^{i}-\frac{3}{4} \phi_{k} \phi^{k} g_{i}^{j}=-T_{i}^{j}
$$

(in geometrized units where $8 \pi G=1$ and $c=1$ ) where $v_{i}=(0,0,0,-1) ; v_{i} v^{i}=-1, \phi_{i}=$ $(0,0,0, \beta(t)), p$ is the isotropic pressure, $\rho$ the matter density, $v^{i}$ the fluid flow vector, and $\beta$ the gauge function. 
The modified Einstein's field equation (2.3) for the metric (2.1) leads to

$$
\begin{aligned}
& \frac{2 b_{44}}{b}+\frac{b_{4}^{2}}{b^{2}}+\frac{1}{b^{2}}-\frac{3}{4} \frac{a^{2}}{b^{4}}+\frac{3}{4} \beta^{2}=-p \\
& \frac{a_{4} b_{4}}{a b}+\frac{b_{44}}{b}+\frac{a_{44}}{a}+\frac{a^{2}}{4 b^{4}}+\frac{3}{4} \beta^{2}=-p, \\
& \frac{2 a_{4} b_{4}}{a b}+\frac{b_{4}^{2}}{b^{2}}+\frac{1}{b^{2}}-\frac{a^{2}}{4 b^{4}}-\frac{3}{4} \beta^{2}=\rho .
\end{aligned}
$$

Equations (2.5) and (2.6) after using barotropic condition $p=\gamma \rho$ lead to

$$
(2 \gamma+1) \frac{a_{4} b_{4}}{a b}+\gamma \frac{b_{4}^{2}}{b^{2}}+\frac{\gamma}{b^{2}}+\frac{b_{44}}{b}+\frac{a_{44}}{a}+(1-\gamma) \frac{a^{2}}{4 b^{4}}+\frac{3}{4}(1-\gamma) \beta^{2}=0
$$

The conservation equation $T_{i ; j}^{j}=0$ leads to

$$
\frac{3}{2} \beta \beta_{4}+\frac{3}{2} \beta^{2}\left(\frac{a_{4}}{a}+\frac{2 b_{4}}{b}\right)=0
$$

which leads to

$$
\beta=\frac{N}{a b^{2}}
$$

$N$ being a constant of integration.

\section{Solution of Field Equations}

For deterministic model, we assume that the shear $(\sigma)$ is proportional to the expansion $(\theta)$. This leads to

$$
a=b^{n}
$$

where $\sigma=\sqrt{2 / 3}\left(a_{4} / a /-b_{4} / b\right), \theta=a_{4} / a+2 b_{4} / b, n$ being a constant.

Equation (3.1) leads to

$$
\begin{gathered}
\frac{a_{4}}{a}=n \frac{b_{4}}{b}, \\
\frac{a_{44}}{a}=\left(n^{2}-n\right)\left(\frac{b_{4}}{b}\right)^{2}+n \frac{b_{44}}{b} .
\end{gathered}
$$


Using (2.9)-(3.3) in (2.7), we have

$$
2 b_{44}+\frac{2\left(2 \gamma n+n^{2}+\gamma\right)}{(n+1)} \frac{b_{4}^{2}}{b}=-\frac{2 \gamma}{b(n+1)}-\frac{(1-\gamma) b^{2 n-3}}{2(n+1)}-\frac{3}{2} \frac{(1-\gamma)}{(n+1)} \frac{N^{2}}{b^{2 n+3}}
$$

To get the simplified result, we assume $n=2$, thus (3.4) leads to

$$
2 b_{44}+\frac{2(5 \gamma+4)}{3} \frac{b_{4}^{2}}{b}=-\frac{2 \gamma}{3 b}-\frac{(1-\gamma)}{6} b-\frac{(1-\gamma)}{2} \frac{N^{2}}{b^{7}}
$$

To find the solution of (3.5), we assume

$$
b_{4}=f(b) .
$$

Thus

$$
b_{44}=f f^{\prime},
$$

where

$$
f^{\prime}=\frac{d f}{d b}
$$

Therefore, (3.5) leads to

$$
\frac{d f^{2}}{d b}+\frac{2(5 \gamma+4)}{3 b} f^{2}=-\frac{2 \gamma}{3 b}-\frac{(1-\gamma)}{6} b-\frac{(1-\gamma)}{2} \frac{N^{2}}{b^{7}}
$$

which again leads to

$$
f^{2}=-\frac{\gamma}{(5 \gamma+4)}-\frac{(1-\gamma)}{4(5 \gamma+7)} b^{2}+\frac{3 N^{2}}{20} b^{-6},
$$

where constant of integration has been assumed zero.

Equation (3.10) leads to

$$
\left(\frac{d b}{d t}\right)^{2}=b^{-6}\left[\frac{3 N^{2}}{20}-\frac{(1-\gamma)}{4(5 \gamma+7)} b^{8}-\frac{\gamma}{5 \gamma+4} b^{6}\right] .
$$

Thus, the metric (2.1) can be written in the form

$$
\begin{aligned}
d s^{2}= & -\frac{T^{6}}{\left[3 N^{2} / 20-((1-\gamma) / 4(5 \gamma+7)) T^{8}-(\gamma /(5 \gamma+4)) T^{6}\right]} d T^{2}+T^{4} d X^{2}+T^{2} d Y^{2} \\
& +\left(T^{2} \sin ^{2} Y+T^{4} \cos ^{2} \Upsilon\right) d Z^{2}-2 T^{4} \cos \Upsilon d X d Z,
\end{aligned}
$$


where $T=b, x=X, y=Y, z=Z$, and cosmic time $t$ is given by

$$
t=\int \frac{T^{3}}{\left[3 N^{2} / 20-((1-\gamma) / 4(5 \gamma+7)) T^{8}-(\gamma /(5 \gamma+4)) T^{6}\right]^{1 / 2}} d T .
$$

\section{Some Physical and Geometrical Properties}

The displacement vector $(\beta)$ is given by (2.9) as

$$
\beta=\frac{N}{a b^{2}}=\frac{N}{T^{4}}
$$

The expansion $(\theta)$ is given by

$$
\theta=\frac{a_{4}}{a}+\frac{2 b_{4}}{b}
$$

which leads to

$$
\theta=\frac{4}{T^{4}}\left[\frac{3 N^{2}}{20}-\frac{(1-\gamma)}{4(5 \gamma+7)} T^{8}-\frac{\gamma T^{6}}{5 \gamma+4}\right]^{1 / 2}
$$

The shear $(\sigma)$ is given by

$$
\sigma^{2}=\frac{2}{3}\left(\frac{a_{4}}{a}-\frac{b_{4}}{b}\right)^{2}
$$

which leads to

$$
\sigma^{2}=\frac{2}{3 T^{8}}\left[\frac{3 N^{2}}{20}-\frac{(1-\gamma)}{4(5 \gamma+7)} T^{8}-\frac{\gamma T^{6}}{5 \gamma+4}\right]
$$

The matter density $(\rho)$ is given by

$$
\rho=\frac{5}{T^{8}}\left[\frac{3 N^{2}}{20}-\frac{(1-\gamma)}{4(5 \gamma+7)} T^{8}-\frac{\gamma T^{6}}{5 \gamma+4}\right]+\frac{1}{T^{2}}-\frac{1}{4}-\frac{3}{4} \frac{N^{2}}{T^{8}}
$$

which leads to

$$
\rho=-\frac{5}{T^{8}}\left[\frac{(1-\gamma)}{4(5 \gamma+7)} T^{8}+\frac{\gamma T^{6}}{(5 \gamma+4)}\right]+\frac{1}{T^{2}}-\frac{1}{4}
$$


which again leads to

$$
\rho=\frac{4}{T^{2}(5 \gamma+4)}-\frac{3}{5 \gamma+7}
$$

and the isotropic pressure is given by

$$
p=\gamma \rho=\gamma\left[\frac{4}{T^{2}(5 \gamma+4)}-\frac{3}{5 \gamma+7}\right] .
$$

The spatial volume $\left(V^{3}\right)$ is given by

$$
V^{3}=T^{4}
$$

\section{Special Case: Dust Model $(p=0)$}

To get the model of dust filled universe, we assume that $n=2$, and using $\gamma=0$ in (3.5), we get

$$
2 b_{44}+\frac{8}{3} \frac{b_{4}^{2}}{b}=-\frac{1}{6} b-\frac{N^{2}}{2 b^{7}}
$$

which leads to

$$
\left(\frac{d b}{d t}\right)^{2}=\frac{3 N^{2}}{20} \frac{1}{b^{6}}-\frac{1}{28} b^{2}
$$

which after integration leads to

$$
b^{2}=\left[\sqrt{\frac{21}{5}} N \sin \left(\frac{2}{\sqrt{7}} \tau\right)\right]^{1 / 2}
$$

where $t+\ell=\tau, \ell$ being constant of integration.

Thus, (2.1) takes the form

$$
\begin{aligned}
d s^{2}= & -d \tau^{2}+\left[\sqrt{\frac{21}{5}} N \sin \left(\frac{2}{\sqrt{7}} \tau\right)\right] d x^{2}+\left[\sqrt{\frac{21}{5}} N \sin \left(\frac{2}{\sqrt{7}} \tau\right)\right]^{1 / 2} d y^{2} \\
& +\left(\left[\sqrt{\frac{21}{5}} N \sin \left(\frac{2}{\sqrt{7}} \tau\right)\right]^{1 / 2} \sin ^{2} y+\left[\sqrt{\frac{21}{5}} N \sin \left(\frac{2}{\sqrt{7}} \tau\right)\right] \cos ^{2} y\right) d z^{2} \\
& -2\left[\sqrt{\frac{21}{5}} N \sin \left(\frac{2}{\sqrt{7}} \tau\right)\right] \cos y d x d z
\end{aligned}
$$


The displacement vector $(\beta)$ is given by (2.9)

$$
\beta=\frac{N}{a b^{2}}=\sqrt{\frac{5}{21}} \operatorname{cosec}\left(\frac{2}{\sqrt{7}} \tau\right) .
$$

The expansion $(\theta)$ is given by

$$
\theta=\frac{a_{4}}{a}+\frac{2 b_{4}}{b}=\frac{2}{\sqrt{7}} \cot \left(\frac{2}{\sqrt{7}} \tau\right) .
$$

The shear $(\sigma)$ is given by

$$
\sigma=\sqrt{\frac{2}{3}}\left(\frac{a_{4}}{a}-\frac{b_{4}}{b}\right)
$$

Thus,

$$
\sigma=\frac{1}{\sqrt{42}} \cot \tau
$$

The matter density $(\rho)$ is given by (2.6)

$$
\rho=\frac{1}{[\sqrt{21 / 5} N \sin ((2 / \sqrt{7}) \tau)]^{1 / 2}}-\frac{3}{7} .
$$

\section{Discussion}

The model (3.12) starts with a big bang at $T=0$, and the expansion in the model decreases as $T$ increases. The displacement vector $(\beta)$ is initially large but decreases due to lapse of time. Since $\sigma / \theta \neq 0$, hence anisotropy is maintained throughout. The reality condition $\rho>0$ implies that the model exists during the span of time given by

$$
T<\sqrt{\frac{4(5 \gamma+7)}{3(5 \gamma+4)}}
$$

The model (3.12) has point type singularity at $T=0$ (MacCallum [24]). The spatial volume increases as $T$ increases.

The model (5.4) starts with a big bang at $\tau=0$, and the expansion in the model decreases as $\tau$ increases. The displacement vector $(\beta)$ is initially large but decreases due to 
lapse of time. Since $\sigma / \theta \neq 0$, hence anisotropy is maintained throughout. The reality condition $\rho>0$ implies that

$$
\sin \left(\frac{2}{\sqrt{7}} \tau\right)<\frac{7 \sqrt{35}}{9 N \sqrt{3}}
$$

where $0<N<1$.

The model has point type singularity at $\tau=0$. (MacCallum [24]).

\section{References}

[1] H. P. Robertson, “Kinematics and World-Structure II," Astrophysical Journal, vol. 83, pp. 187-201, 1936.

[2] W. De Sitter, "On Einstein's theory of gravitation, and its astronomical consequences," Monthly Notices of the Royal Astronomical Society, vol. 78, pp. 3-28, 1917.

[3] A. H. Taub, "Empty space-times admitting a three parameter group of motions," Annals of Mathematics. Second Series, vol. 53, pp. 472-490, 1951.

[4] E. Newman, L. Tamburino, and T. Unti, "Empty-space generalization of the Schwarzschild metric," Journal of Mathematical Physics, vol. 4, no. 7, pp. 915-923, 1963.

[5] P. C. Vaidya and L. K. Patel, "Gravitational fields with space-times of Bianchi type IX," Pramana, vol. 27, no. 1-2, pp. 63-72, 1986.

[6] K. D. Krori, T. Chaudhury, C. R. Mahanta, and A. Mazumdar, "Some exact solutions in string cosmology," General Relativity and Gravitation, vol. 22, no. 2, pp. 123-130, 1990.

[7] S. Chakraborty and G. C. Nandy, "Cosmological studies in Bianchi II, VIII space-time," Astrophysics and Space Science, vol. 198, no. 2, pp. 299-308, 1992.

[8] S. Chakraborty, "A study on Bianchi-IX cosmological model," Astrophysics and Space Science, vol. 180, no. 2, pp. 293-303, 1991.

[9] R. Bali and R. D. Upadhaya, "Bianchi type IX string dust cosmological model in general relativity," Proceedings of the National Academy of Sciences. India. Section A, vol. 73, no. 2, pp. 239-247, 2003.

[10] H. Weyl, "Gravitation and Elektricität," Sber. Preuss. Akad. d. Wisson Chaften, vol. 465, 1918.

[11] G. Lyra, “Über eine Modifikation der Riemannschen Geometrie," Mathematische Zeitschrift, vol. 54, pp. 52-64, 1951.

[12] D. K. Sen, “A static cosmological model," Zeitschrift für Physik, vol. 149, no. 3, pp. 311-323, 1957.

[13] W. D. Halford, "Scalar-tensor theory of gravitation in a Lyra manifold," Journal of Mathematical Physics, vol. 13, no. 11, pp. 1699-1703, 1972.

[14] T. Singh and G. P. Singh, "Bianchi type-I cosmological models in Lyra's geometry," Journal of Mathematical Physics, vol. 32, no. 9, pp. 2456-2458, 1991.

[15] F. Rahaman and J. K. Bera, "Higher dimensional cosmological model in Lyra geometry," International Journal of Modern Physics D, vol. 10, no. 5, pp. 729-733, 2001.

[16] F. Rahaman, N. Begum, G. Bag, and B. C. Bhui, "Cosmological models with negative constant deceleration parameter in Lyra geometry," Astrophysics and Space Science, vol. 299, no. 3, pp. 211-218, 2005.

[17] A. Pradhan, V. K. Yadav, and I. Chakrabarty, "Bulk viscous FRW cosmology in Lyra geometry," International Journal of Modern Physics D, vol. 10, no. 3, pp. 339-349, 2001.

[18] A. Pradhan, I. Aotemshi, and G. P. Singh, "Plane symmetric domain wall in Lyra geometry," Astrophysics and Space Science, vol. 288, no. 3, pp. 315-325, 2003.

[19] A. Pradhan, L. Yadav, and A. K. Yadav, "Isotropic homogeneous universe with a bulk viscous fluid in Lyra geometry," Astrophysics and Space Science, vol. 299, no. 1, pp. 31-42, 2005.

[20] R. Bali and N. K. Chandnani, "Bianchi type-I cosmological model for perfect fluid distribution in Lyra geometry," Journal of Mathematical Physics, vol. 49, no. 3, Article ID 032502, 2008.

[21] R. Bali and N. K. Chandnani, "Bianchi type-III bulk viscous dust filled universe in Lyra geometry," Astrophysics and Space Science, vol. 318, no. 3-4, pp. 225-230, 2008.

[22] S. Ram, M. Zeyauddin, and C. P. Singh, "Bianchi type V cosmological models with perfect fluid and heat conduction in lyra's geometry," International Journal of Modern Physics A, vol. 23, no. 31, pp. 49915005, 2008. 
[23] R. Bali, N. K. Chandnani, and J. P. Dhanka, "Bianchi type I magnetized dust filled universe in Lyra geometry," International Journal of Modern Physics A, vol. 25, no. 25, pp. 4839-4848, 2010.

[24] M. A.H. MacCallum, "A class of homogeneous cosmological models III: asymptotic behaviour," Communications in Mathematical Physics, vol. 20, no. 1, pp. 57-84, 1971. 


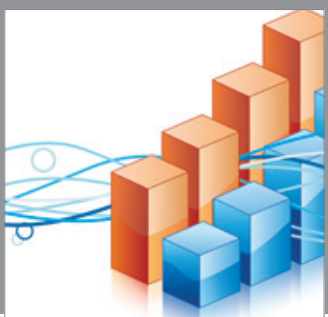

Advances in

Operations Research

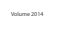

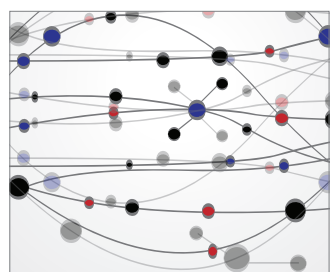

\section{The Scientific} World Journal
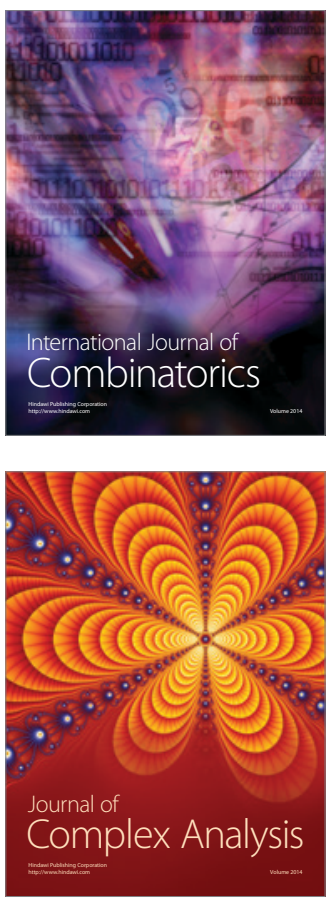

International Journal of

Mathematics and

Mathematical

Sciences
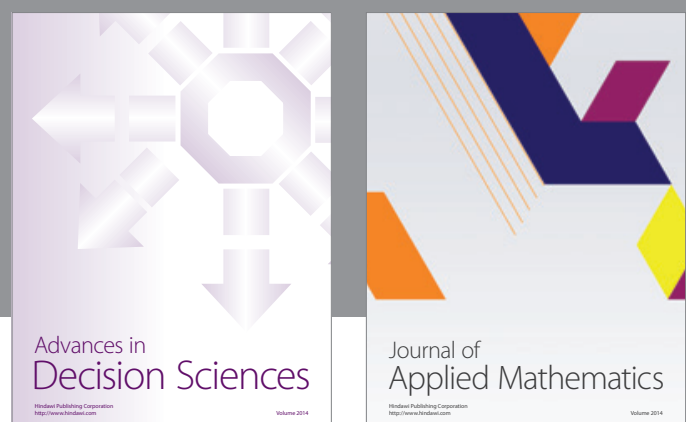

Journal of

Applied Mathematics
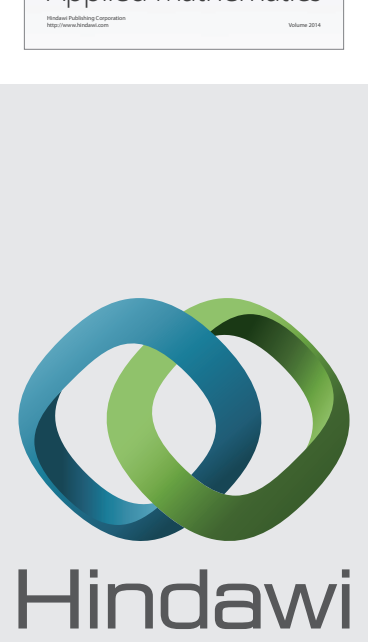

Submit your manuscripts at http://www.hindawi.com
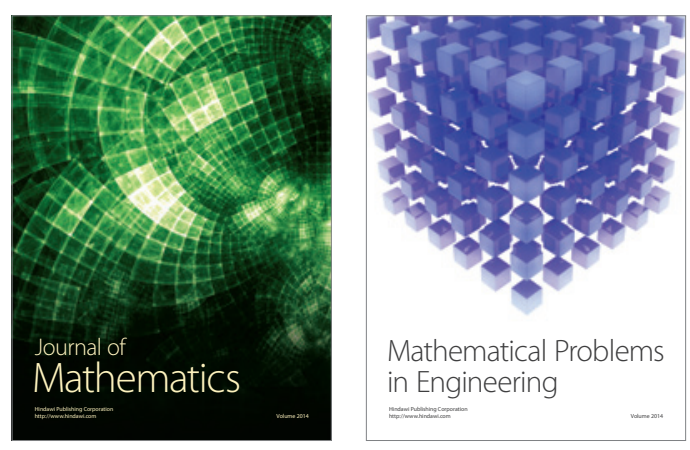

Mathematical Problems in Engineering
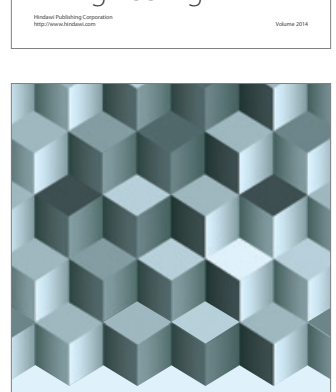

Journal of

Function Spaces
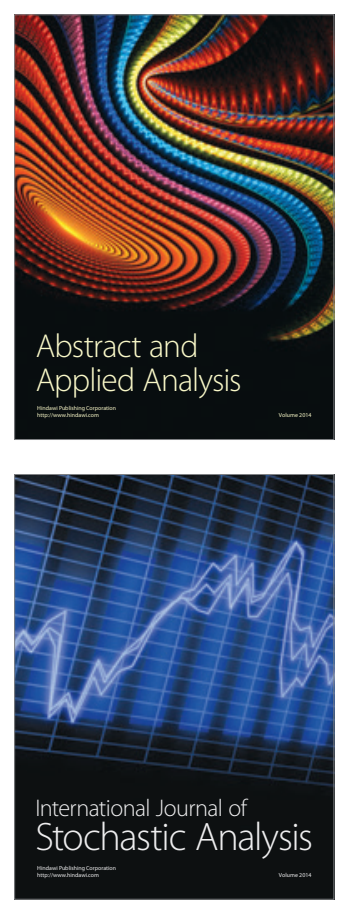

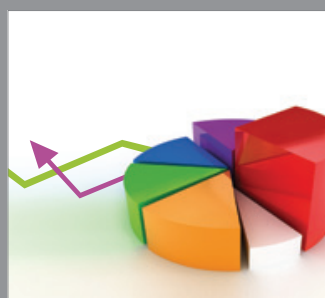

ournal of

Probability and Statistics

Promensencen
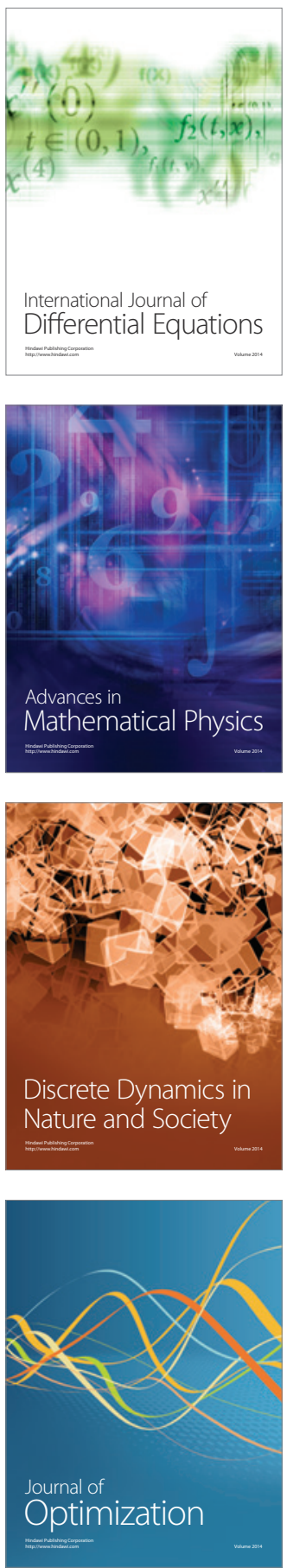